\title{
VOLUME ESTIMATES AND CLASSIFICATION THEOREM FOR CONSTANT WEIGHTED MEAN CURVATURE HYPERSURFACES
}

\author{
SAUL ANCARI AND IGOR MIRANDA
}

\begin{abstract}
In this paper, we prove a classification for complete embedded constant weighted mean curvature hypersurfaces $\Sigma \subset \mathbb{R}^{n+1}$. We characterize the hyperplanes and generalized round cylinders by using an intrinsic property on the norm of the second fundamental form. Furthermore, we prove an equivalence of properness, finite weighted volume and exponential volume growth for submanifolds with weighted mean curvature of at most linear growth.
\end{abstract}

\section{INTRODUCTION}

In the mean curvature flow theory, one of the main problems is to understand possible singularities that the flow goes through. Self-shrinkers play an important role in this theory since they are singularity models for the flows . These hypersurfaces satisfy the following mean curvature condition

$$
H=\frac{\langle x, \nu\rangle}{2}
$$

where $H, x$ and $\nu$ stand for the mean curvature of $\Sigma$, the position vector in $\mathbb{R}^{n}$ and the unit normal vector of $\Sigma$, respectively. Another characterization of the self-shrinkers is that they are critical points of the weighted area functional

$$
F(\Sigma)=\int_{\Sigma} e^{-\frac{|x|^{2}}{4}} d v
$$

There is a great interest in studying two-sided smooth hypersurfaces $\Sigma \subset \mathbb{R}^{n+1}$ which are critical points of the functional (1.1) for variations $G:(-\varepsilon, \varepsilon) \times \Sigma \rightarrow \mathbb{R}^{n+1}$ that preserve enclosed weighted volume. These variations can be represented by functions $u: \Sigma \rightarrow \mathbb{R}$ defined by

$$
u(x)=\left\langle\partial_{t} G(0, x), \nu(x)\right\rangle
$$

such that $\int_{\Sigma} u e^{-|x|^{2} / 4} d v=0$, where $\nu$ is the normal vector of $\Sigma$. It is well known that these hypersurfaces satisfy the following condition

$$
H=\frac{\langle x, \nu\rangle}{2}+\lambda
$$

where $\lambda \in \mathbb{R}$. Such hypersurfaces are known as constant weighted mean curvature hypersurfaces. Throughout this paper, whenever $\Sigma$ satisfies the mean curvature condition above, they will be called CWMC hypersurfaces and $\lambda$ denotes the weighted mean curvature. The study of such hypersurfaces arises in geometry and probability as solutions to the Gaussian isoperimetric problem.

Here are some examples of CWMC hypersurfaces.

Example 1.1. Any self-shrinker is a CWMC hypersurface with $\lambda=0$.

Example 1.2. All hyperplanes in $\mathbb{R}^{n+1}$ are CWMC hypersurfaces with $\lambda= \pm \frac{d}{2}$, where $d$ denotes the distance from the hyperplane to the origin and the sign depends on the orientation. Indeed, let $\Sigma \subset \mathbb{R}^{n+1}$ be a hyperplane and $x_{0} \in \Sigma$ such that $d(\Sigma, 0)=d\left(x_{0}, 0\right)=d$. This implies that $\pm d=\left\langle x_{0}, \nu\right\rangle$,

Key words and phrases. weighted mean curvature, exponential volume growth, finite weighted volume.

This study was financed in part by the Coordenao de Aperfeioamento de Pessoal de Nvel Superior - Brasil (CAPES) Finance Code 001 . 
where $\nu$ is the normal vector of $\Sigma$. Since $\Sigma=\left\{x \in \mathbb{R}^{n+1} ;\langle x, \nu\rangle= \pm d\right\}$ and the mean curvature of $\Sigma$ vanishes, we have

$$
H=0=\frac{\langle x, \nu\rangle}{2} \pm \frac{d}{2}
$$

Therefore, $\Sigma$ is a CWMC hypersurface with $\lambda= \pm d / 2$, as asserted.

Example 1.3. The spheres centered at the origin with radius $\sqrt{\lambda^{2}+2 n}-\lambda$ are CWMC hypersurfaces in $\mathbb{R}^{n+1}$.

Example 1.4. The cylinders $S_{r}^{k}(0) \times \mathbb{R}^{n+k}$ with radius $\sqrt{\lambda^{2}+2 k}-\lambda$ are also CWMC hypersurfaces in $\mathbb{R}^{n+1}$.

In [Hui90], Huisken proved that the spheres $S^{n}(\sqrt{2 n})$ are the only compact self-shrinkers with nonnegative mean curvature of dimension $n \geq 2$. Huisken [Hui] also showed that the generalized cylinders are the only complete self-shrinkers in $\mathbb{R}^{n+1}$ with non-negative mean curvature, polynomial volume growth and such that the norm of the second fundamental form is bounded. Colding and Minicozzi CM12 generalized Huisken's classification by removing the boundness condition on the second fundamental form. Rimoldi Rim14 proved the same classification theorem as Colding-Minicozzi, replacing the polynomial volume growth by a assumption on the integrability of $|A|$. More specifically

Theorem 1.1. $S^{k}(\sqrt{2 n}) \times \mathbb{R}^{n-k}, 0 \leq k \leq n$, are the only smooth complete embedded self-shrinkers in $\mathbb{R}^{n+1}$ with $H \geq 0$ and $|A| \in L_{f}^{2}(\Sigma)$.

Recently, there has been much interest around classification results for CWMC hypersurfaces. For instance, Guang Gua18 proved a gap theorem for CWMC hypersurfaces, showing that if the hypersurface has a bound condition on $|A|$, then it must be a generalized cylinder. In [Hei17], Heilman proved a generalization of Colding-Minicozzi theorem for $\lambda>0$. For what follows, it is important to recall a result obtained by Cheng and Wei [CW18] for CWMC hypersurfaces. More precisely, they establish the following result.

Theorem 1.2. $S^{k} \times \mathbb{R}^{n-k}, 0 \leq k \leq n$, are the only complete embedded CWMC hypersurfaces with polynomial volume growth in $\mathbb{R}^{n+1}$ satisfying $H-\lambda \geq 0$ and $\lambda\left(\operatorname{tr} A^{3}(H-\lambda)+\frac{|A|^{2}}{2}\right) \leq 0$.

The hypothesis of polynomial volume growth is used to show that weighted integrals converge in order to justify integration by parts. In fact this hypothesis also implies the hypersurface is proper. In this paper, we will replace the polynomial volume growth assumption considered by Cheng-Wei by an intrinsic condition. More specifically, we prove the following result.

Theorem 1.3. Let $\Sigma \subset \mathbb{R}^{n+1}$ be a complete embedded CWMC hypersurface. Suppose that $\Sigma$ satisfies the following properties:

(i) $H-\lambda \geq 0$;

(ii) $\lambda\left(\operatorname{tr} A^{3}(H-\lambda)+\frac{|A|^{2}}{2}\right) \leq 0$;

(iii) $\frac{1}{k^{2}} \int_{B_{2 k}^{\Sigma}(p) \backslash B_{k}^{\Sigma}(p)}|A|^{2} e^{-f} \rightarrow 0$, when $k \rightarrow \infty$, for a fixed point $p \in \Sigma$.

Then $\Sigma$ must be either a hyperplane or $S_{r}^{k}(0) \times \mathbb{R}^{n-k}, 1 \leq k \leq n$.

Remark 1.1. Note that Theorem 1.3 generalizes Theorem 1.1 proved by Rimoldi.

The second result of this paper provides a relation between the exponential volume growth condition and properness for submanifolds with weighted mean curvature of at most linear growth. For selfshrinkers, Ding and Xin $\left[\mathrm{DX}^{+} 13\right.$, proved that the properness condition implies Euclidean volume growth. Cheng and Zhou [CZ13] proved that properness, Euclidean volume growth, polynomial volume growth and finite weighted volume are all equivalent for self-shrinkers. Alencar and Rocha [AR18] showed that $\left|\vec{H}_{f}\right|<\infty$ and finite weighted volume imply properness (see section 2 for the definition of $\vec{H}_{f}$ ). Also, a consequence from one of their results is that for $f=|x|^{2} / 4, \sup \left\langle\overrightarrow{H_{f}}, \bar{\nabla} f\right\rangle<\infty$ and 
properness imply finite weighted volume and polynomial volume growth. Recently, Cheng, Vieira and Zhou [CVZ19] proved that for hypersurfaces in $\mathbb{R}^{n+1}$ with the norm of the weighted mean curvature bounded, properness is equivalent to polynomial volume growth. Here is our second result.

Theorem 1.4. For any complete n-dimensional immersed submanifold $\Sigma$ in $\mathbb{R}^{n+p}, p \geq 1$, satisfying

$$
\left|\vec{H}_{f}\right| \leq a_{1} r+a_{0} \text { on } \Sigma \cap B_{r}(0), \forall r>0
$$

for some $a_{0}, a_{1} \geq 0$, where $f=|x|^{2} / 4$, the following statements are equivalent:

(i) $\Sigma$ properly immersed on $\mathbb{R}^{n+p}$;

(ii) There exist constants $C, \bar{a}_{0}, \bar{a}_{1}, \bar{a}_{2}$, with $\bar{a}_{2}<\frac{1}{4}$, such that

$$
V\left(B_{r}(0) \cap \Sigma\right) \leq C e^{\bar{a}_{2} r^{2}+\bar{a}_{1} r+\bar{a}_{0}} ;
$$

(iii) $\int_{\Sigma} e^{-f}<\infty$.

Remark 1.2. Recall that a submanifold $\Sigma^{n} \subset \mathbb{R}^{n+p}$ that satisfies $\vec{H}=\frac{x^{\perp}}{2}$ is called self-expander. A translating soliton is a submanifold in $\mathbb{R}^{n+p}$ that satisfies $\vec{H}=z^{\perp}$, for $z \in \mathbb{R}$ fixed. Note that for self-expanders $\left|\vec{H}_{f}\right|=\left|x^{\perp}\right|$ and for translating solitons $\left|\vec{H}_{f}\right|=\left|z^{\perp}+\frac{x^{\perp}}{2}\right|$. Therefore, if $\Sigma^{n} \subset$ $\mathbb{R}^{n+p}$ is a complete properly immersed self-expander or translating soliton, then there exist constants $C, \bar{a}_{0}, \bar{a}_{1}, \bar{a}_{2}$, with $\bar{a}_{2}<\frac{1}{4}$, such that

$$
V\left(B_{r}(0) \cap \Sigma\right) \leq C e^{\bar{a}_{2} r^{2}+\bar{a}_{1} r+\bar{a}_{0}} .
$$

Finally, a corollary of Theorem 1.3 and Theorem 1.4 is the following.

Corollary 1.5. Let $\Sigma \subset \mathbb{R}^{n+1}$ be a complete properly embedded CWMC hypersurface. If $H-\lambda \geq 0$ and $\lambda\left(\operatorname{tr} A^{3}(H-\lambda)+\frac{|A|^{2}}{2}\right) \leq 0$, then $\Sigma$ must be a hyperplane or $S_{r}^{k}(0) \times \mathbb{R}^{n-k}, 1 \leq k \leq n$.

This work is divided into four sections. In section 2, we recall some notations, basic tools and key formulas for CWMC hypersurfaces. In section 3, we will prove the classification theorem. In section 4. we will prove the equivalence between exponential volume growth and properness. In section 5, we provide an application of the main results of this paper.

Acknowledgement: The authors would like to thank professor Detang Zhou for his support, suggestions and encouragement throughout this work. We also want to thank professor Ernani Ribeiro Jr. for his helpful advices.

\section{Preliminaries}

In this section, we will establish some notations and recall some definitions and basic results.

Let us denote by $\left(\bar{M}^{n}, \bar{g}, e^{-f} d v\right)$ a smooth measure metric space, which is a n-dimensional Riemannian manifold $(\bar{M}, \bar{g})$ endowed with $e^{-f} d v$ volume form, where $f$ is a smooth function over $\bar{M}$ and $d v$ is the volume form induced by the metric $\bar{g}$. Throughout this work, whenever we integrate, we will omit $d v$. We will also denote the connection of $(\bar{M}, \bar{g})$ by $\bar{\nabla}$.

For what follows, recall that the drifted Laplacian over $(\bar{M}, \bar{g})$ is

$$
\bar{\Delta}_{f} u=\bar{\Delta} u-\langle\bar{\nabla} f, \bar{\nabla} u\rangle \text {. }
$$

If $\Sigma \subset \mathbb{R}^{n+1}$ is a hypersurface and $f=|x|^{2} / 4$, we denote the drifted Laplacian by

$$
\mathcal{L}=\Delta-\frac{1}{2}\langle x, \nabla\rangle \text {. }
$$

This operator is self-adjoint over $L^{2}\left(\Sigma, g, e^{-f} d \sigma\right)$. More precisely, 
Lemma 2.1. If $\Sigma \subset \mathbb{R}^{n+1}, u \in C_{0}^{1}(\Sigma)$ and $v \in C^{2}(\Sigma)$, then

$$
\int_{\Sigma} u \mathcal{L} v e^{-f}=-\int_{\Sigma}\langle\nabla u, \nabla v\rangle e^{-f}
$$

Proof. For a detailed proof, see [CM12].

Let $\Sigma$ be a submanifold immersed on $\bar{M}$, endowed with the metric g induced by $\bar{g}$. We will denote by $\nabla, \Delta$ and $d \sigma$, the connection, Laplacian and volume form, respectively. The second fundamental form of $(\Sigma, g)$ at $p \in \Sigma$ is defined as

$$
A(X, Y)=\left(\bar{\nabla}_{X} Y\right)^{\perp},
$$

where $X, Y \in T_{p} \Sigma$. The mean curvature vector $\vec{H}$ of $\Sigma$ at $p$ is defined as

$$
\vec{H}=\operatorname{tr} A \text {. }
$$

The weighted mean curvature vector of $\Sigma$ at $p$ is defined as

$$
\overrightarrow{H_{f}}=\vec{H}+(\bar{\nabla} f)^{\perp} .
$$

If $\Sigma$ is a hypersurface immersed in $\bar{M}^{n+1}$, we will denote by $h_{i j}=\left\langle A\left(e_{i}, e_{j}\right), \nu\right\rangle$, where $\left\{e_{i}\right\}$ is an orthonormal basis of $T_{p} \Sigma$. The mean curvature of $\Sigma$ is defined as $\vec{H}=-H \nu$ and the weighted mean curvature as $\overrightarrow{H_{f}}=-H_{f} \nu$. Throughout this work, whenever $\Sigma \subset \mathbb{R}^{n+p}$, we will be considering the smooth measure metric space $\left(\mathbb{R}^{n+p}, g, e^{-f} d \sigma\right)$, with $f=\frac{|x|^{2}}{4}$. A hypersurface $\Sigma \subset \mathbb{R}^{n+1}$ is CWMC hypersurface if

$$
H_{f}=\lambda \text {. }
$$

In particular, if $\lambda=0, \Sigma$ is a self-shrinker.

For CWMC hypersurfaces, the following equations will be needed to prove the classification theorem.

Lemma 2.2. If $\Sigma \subset \mathbb{R}^{n+1}$ is a CWMC hypersurface, then

$$
\mathcal{L}(H-\lambda)+\left(|A|^{2}-\frac{1}{2}\right)(H-\lambda)=\frac{\lambda}{2}
$$

and

$$
\mathcal{L}|A|+\left(|A|^{2}-\frac{1}{2}\right)|A|=\frac{|\nabla A|^{2}-|\nabla| A||^{2}}{|A|}-\frac{\lambda \operatorname{tr} A^{3}}{|A|} .
$$

Proof. For a detailed proof, see Gua18].

\section{A Classification for CWMC hypersurfaces}

In this section, we prove Theorem 1.3. The main idea of proving this theorem is to use a technique similar to that used by Tasayco and Zhou in [TZ17] to prove that $|A|=C(H-\lambda)$, where $\mathrm{C}$ is a constant.

Proof of Theorem 1.3. For $\lambda \leq 0$, from Lemma 2.2 we have

$$
\mathcal{L}(H-\lambda)+\left(|A|^{2}-\frac{1}{2}\right)(H-\lambda)=\frac{\lambda}{2} \leq 0 .
$$

Since $H-\lambda \geq 0$, by the maximum principle we can conclude that either $H-\lambda=0$ or $H-\lambda>0$. If $H-\lambda=0$, then from Lemma 2.2 we conclude that $\lambda=0$, which implies that $\Sigma$ is a self-shrinker. Moreover, Colding-Minicozzi proved in [CM12] that a self-shrinker such that $H=0$ has to be a hyperplane. If $\lambda>0$ and $H-\lambda=0$ at some point $p \in \Sigma$, from hypothesis (ii)

$$
0 \geq \lambda\left(\operatorname{tr} A^{3}(H-\lambda)+\frac{|A|^{2}}{2}\right)=\frac{\lambda|A|^{2}}{2}
$$


at $p \in \Sigma$. This implies that $|A|(p)=0$, but this contradicts the fact that $H(p)>0$. Finally, we only we need to consider the case $H-\lambda>0$.

By adapting Tasayco-Zhou's lemma [TZ17], we will demonstrate that either $|A|=0$ or $|A|=$ $C(H-\lambda)$, for $C>0$. Consider the functions $u=H-\lambda$ and $v=\sqrt{|A|^{2}+\varepsilon}$. Computing $\mathcal{L}(u)$ and $\mathcal{L}(v)$, we get

$$
\mathcal{L} u+\left(|A|^{2}-\frac{1}{2}\right) u=\frac{\lambda}{2}
$$

and

Since

$$
\mathcal{L} v+\left(|A|^{2}-\frac{1}{2}\right) v=\frac{|\nabla A|^{2}-|\nabla v|^{2}}{v}+\left(|A|^{2}-\frac{1}{2}\right) \frac{\varepsilon}{v}-\frac{\lambda \operatorname{tr} A^{3}}{v} .
$$

Let us consider $w=\frac{v}{u}$. Thus, from (3.1) we get

$$
\frac{|\nabla A|^{2}-|\nabla v|^{2}}{v} \geq 0, \quad \mathcal{L} v+\left(|A|^{2}-\frac{1}{2}\right) v \geq-\frac{\varepsilon}{2 v}-\frac{\lambda \operatorname{tr} A^{3}}{v} .
$$

$$
\begin{aligned}
\mathcal{L} v & =w \mathcal{L} u+2\langle\nabla w, \nabla u\rangle+u \mathcal{L} w \\
& =w\left(\frac{\lambda}{2}-|A|^{2}+\frac{1}{2}\right)+2\langle\nabla w, \nabla u\rangle+u \mathcal{L} w .
\end{aligned}
$$

By (3.2), we have

$$
u \mathcal{L} w \geq-\frac{1}{v}\left(\frac{\varepsilon}{2}+\lambda \operatorname{tr} A^{3}\right)-\frac{\lambda w}{2}-2\langle\nabla w, \nabla u\rangle
$$

Using hypothesis (ii), it is possible to conclude that

$$
\frac{u}{v}\left(-\frac{\varepsilon}{2}-\lambda \operatorname{tr} A^{3}\right)-\frac{v \lambda}{2} \geq-\frac{\varepsilon H}{2 v} .
$$

From the inequality above, we obtain

$$
\mathcal{L} w \geq-\frac{\varepsilon H}{2 v u^{2}}-2\langle\nabla w, \nabla \log u\rangle .
$$

For a function $\varphi \in C_{0}^{\infty}(\Sigma)$, using integration by parts and (3.4), we get

$$
\begin{aligned}
\int_{\Sigma} \varphi^{2}|\nabla w|^{2} e^{-f} & =-\int_{\Sigma} \varphi^{2} w \mathcal{L} w e^{-f}-\int_{\Sigma} 2 \varphi w\langle\nabla \varphi, \nabla w\rangle e^{-f} \\
& \leq 2 \int_{\Sigma} \varphi^{2} w\langle\nabla w, \nabla \log u\rangle e^{-f}+\frac{\varepsilon}{2} \int_{\Sigma} \frac{\varphi^{2} w H}{v u^{2}} e^{-f}-\int_{\Sigma} 2 \varphi w\langle\nabla \varphi, \nabla w\rangle e^{-f} \\
& =2 \int_{\Sigma}\langle\varphi \nabla w, \varphi w \nabla \log u-w \nabla \varphi\rangle e^{-f}+\frac{\varepsilon}{2} \int_{\Sigma} \frac{\varphi^{2} H}{u^{3}} e^{-f} \\
& \leq \frac{1}{2} \int_{\Sigma} \varphi^{2}|\nabla w|^{2} e^{-f}+2 \int_{\Sigma} w^{2}|\varphi \nabla \log u-\nabla \varphi|^{2} e^{-f}+\frac{\varepsilon}{2} \int_{\Sigma} \frac{\varphi^{2} H}{u^{3}} e^{-f} .
\end{aligned}
$$

Therefore,

$$
\int_{\Sigma} \varphi^{2}|\nabla w|^{2} \leq 4 \int_{\Sigma} w^{2}|\varphi \nabla \log u-\nabla \varphi|^{2} e^{-f}+\varepsilon \int_{\Sigma} \frac{\varphi^{2} H}{u^{3}} e^{-f} .
$$

Choosing $\varphi=\psi u, \psi \in C_{0}^{\infty}(\Sigma)$, we have

$$
\int_{\Sigma} \psi^{2} u^{2}|\nabla w|^{2} e^{-f} \leq 4 \int_{\Sigma} v^{2}|\nabla \psi|^{2} e^{-f}+\varepsilon \int_{\Sigma} \psi^{2} e^{-f}+\varepsilon \lambda \int_{\Sigma} \frac{\psi^{2}}{u} e^{-f} .
$$

For $\lambda \geq 0$, choosing $\varepsilon=0$ we obtain

$$
\int_{\Sigma} \psi^{2} u^{2}|\nabla w|^{2} e^{-f} \leq 4 \int_{\Sigma} v^{2}|\nabla \psi|^{2} e^{-f}
$$


Consider a sequence $\psi_{k} \in C_{0}^{\infty}(\Sigma)$, such that $\psi_{k}=1$ in $B_{k}^{\Sigma}(p), \psi_{k}=0$ in $\Sigma \backslash B_{2 k}^{\Sigma}(p)$ and $\left|\nabla \psi_{k}\right| \leq 1 / k$ for every $k$, we have

$$
\begin{aligned}
\int_{\Sigma} \psi_{k}^{2} u^{2}|\nabla w|^{2} e^{-f} & \leq 4 \int_{B_{2 k}^{\Sigma}(p) \backslash B_{k}^{\Sigma}(p)} v^{2}\left|\nabla \psi_{k}\right|^{2} e^{-f} \\
& \leq \frac{4}{k^{2}} \int_{B_{2 k}^{\Sigma}(p) \backslash B_{k}^{\Sigma}(p)} v^{2} e^{-f} \\
& =\frac{4}{k^{2}} \int_{B_{2 k}^{\Sigma}(p) \backslash B_{k}^{\Sigma}(p)}|A|^{2} e^{-f} .
\end{aligned}
$$

By the monotone convergence theorem and hypothesis (iii), we get

$$
\int_{\Sigma} u^{2}\left|\nabla\left(\frac{|A|}{H-\lambda}\right)\right|^{2} e^{-f}=0
$$

which implies that $|A|=C(H-\lambda)$, for a constant $C>0$.

For $\lambda<0$, we have

$$
\int_{\Sigma} \psi^{2} u^{2}|\nabla w|^{2} e^{-f} \leq 4 \int_{\Sigma} v^{2}|\nabla \psi|^{2} e^{-f}+\varepsilon \int_{\Sigma} \psi^{2} e^{-f}
$$

As in the other case, consider a sequence $\psi_{k} \in C_{0}^{\infty}(\Sigma)$, such that $\psi_{k}=1$ in $B_{k}^{\Sigma}(p), \psi_{k}=0$ in $\Sigma \backslash B_{2 k}^{\Sigma}(p)$ and $\left|\nabla \psi_{k}\right| \leq 1 / k$ for every $k$, hence we get

$$
\begin{aligned}
\int_{\Sigma} \psi_{k}^{2} u^{2}|\nabla w|^{2} e^{-f} & \leq 4 \int_{\Sigma} v^{2}\left|\nabla \psi_{k}\right|^{2} e^{-f}+\varepsilon \int_{\Sigma} \psi_{k}^{2} e^{-f} \\
& \leq \frac{4}{k^{2}} \int_{B_{2 k}^{\Sigma}(p) \backslash B_{k}^{\Sigma}(p)}|A|^{2} e^{-f}+\frac{4 \varepsilon}{k^{2}} \int_{B_{2 k}^{\Sigma}(p) \backslash B_{k}^{\Sigma}(p)} e^{-f}+\varepsilon \int_{B_{2 k}^{\Sigma}(p)} e^{-f} .
\end{aligned}
$$

Choosing $\varepsilon=\left(k \int_{B_{2 k}^{\Sigma}(p)} e^{-f}\right)^{-1}$, we have

$$
\int_{\Sigma} \psi_{k}^{2} u^{2}|\nabla w|^{2} e^{-f} \leq \frac{4}{k^{2}} \int_{B_{2 k}^{\Sigma}(p) \backslash B_{k}^{\Sigma}(p)}|A|^{2} e^{-f}+\frac{4}{k^{3}}+\frac{1}{k} .
$$

Hence, by hypothesis (iii)

$$
\lim _{k \rightarrow \infty} \int_{\Sigma} \psi_{k}^{2} u^{2}|\nabla w|^{2} e^{-f}=0
$$

If the set

$$
\mathcal{A}=\{p \in \Sigma ;|A|(p)=0\}
$$

is not empty, consider $\mathcal{B}=\Sigma \backslash \mathcal{A}$. Since $\mathcal{B}$ is an open set, let $p \in \mathcal{B}$ and $B_{p}^{\Sigma}(r) \subset \mathcal{B}$. For k sufficiently large, $B_{p}^{\Sigma}(r) \subset \operatorname{supp} \psi_{k}$ and $\psi_{k}=1$ in $B_{p}^{\Sigma}(r)$. Hence

$$
\lim _{k \rightarrow \infty} \int_{B_{p}^{\Sigma}(r)} u^{2}|\nabla w|^{2} e^{-f}=0
$$

By the dominated convergence theorem, we conclude that $|A| /(H-\lambda)$ is constant in $B_{p}^{\Sigma}(r)$. Since p is arbitrary, it is possible to conclude that $|A| /(H-\lambda)$ is constant in $\mathcal{B}$. Since $\mathcal{A} \neq \emptyset$, using a continuity argument, we conclude that $|A|=0$. If $\mathcal{A}=\emptyset$, by the dominated convergence theorem

$$
\int_{\Sigma} u^{2}\left|\nabla\left(\frac{|A|}{H-\lambda}\right)\right|^{2} e^{-f}=0
$$

which implies $|A|=C(H-\lambda)$ for a constant $C>0$. Hence, when $H-\lambda>0$, we conclude that either $|A|=0$ or $|A|=C(H-\lambda)$ for a constant $C>0$. If $|A|=0$, then $\Sigma$ is a hyperplane. Otherwise, since 
$|A|=C(H-\lambda)$

$$
\begin{aligned}
\mathcal{L}|A| & =\frac{|A|}{H-\lambda} \mathcal{L}(H-\lambda) \\
& =\frac{|A| \lambda}{2(H-\lambda)}+\left(\frac{1}{2}-|A|^{2}\right)|A| .
\end{aligned}
$$

On the other hand

$$
\mathcal{L}|A|=\left(\frac{1}{2}-|A|^{2}\right)|A|+\frac{|\nabla A|^{2}-\left.|\nabla| A\right|^{2}}{|A|}-\frac{\lambda \operatorname{tr} A^{3}}{|A|}
$$

Hence, from the equations above we have

$$
\begin{aligned}
\frac{|\nabla A|^{2}-\left.|\nabla| A\right|^{2}}{|A|} & =\frac{|A| \lambda}{2(H-\lambda)}+\frac{\lambda \operatorname{tr} A^{3}}{|A|} \\
& =\frac{\lambda}{(H-\lambda)|A|}\left(\operatorname{tr} A^{3}(H-\lambda)+\frac{|A|^{2}}{2}\right) .
\end{aligned}
$$

Using the hypothesis $\lambda\left(\operatorname{tr} A^{3}(H-\lambda)+\frac{|A|^{2}}{2}\right) \leq 0$ and the equality above, we conclude that

$$
|\nabla| A||=|\nabla A| .
$$

Fixing $p \in \Sigma$ and $\left\{E_{i}\right\}_{1 \leq i \leq n}$ a orthonormal basis for $T_{p} \Sigma$, (3.5) implies that for each $k$ there exists a constant $C_{k}$ such that

$$
h_{i j k}=C_{k} h_{i j}
$$

for all $i, j$. Considering a base such that $h_{i j}=\lambda_{i} \delta_{i j}$, by the Codazzi equation, we have

$$
h_{i j k}=0
$$

unless $i=j=k$. If $\lambda_{i} \neq 0$ and $i \neq j$ then

$$
0=h_{i i j}=C_{j} \lambda_{i}
$$

It follows that $C_{j}=0$. Hence, if the rank of the matrix $\left(h_{i j}\right)$ is at least two at p, then $\nabla A(p)=0$. To show that $\nabla A=0$, let us fix $q \in \Sigma$ and suppose that $\lambda_{1}(q)$ and $\lambda_{2}(q)$ are the largest eigenvalues of $\left(h_{i j}\right)(q)$. Define the following set

$$
\Lambda=\left\{q \in \Sigma ; \lambda_{1}(q)=\lambda_{1}(p), \lambda_{2}(q)=\lambda_{2}(p)\right\} .
$$

Using the continuity of the $\lambda_{i}^{\prime} s$, it is possible to prove that the set $\Lambda$ is open and closed. Since $p \in \Lambda$ and $\Sigma$ is connected, $\Lambda=\Sigma$. Therefore, $\nabla A=0$ everywhere on $\Sigma$. Hence, $\Sigma$ is a isoparametric hypersurface and by a theorem proved by Lawson in [Law69], $\Sigma$ must be $S^{k}(r) \times \mathbb{R}^{n-k}$ with $2 \leq k \leq n$.

If the rank of the matrix $\left(h_{i j}\right)$ is one, then

$$
H^{2}=|A|^{2}=C^{2}(H-\lambda)^{2} .
$$

From this equation, $H$ must be constant. Moreover, from

$$
|\nabla A|=|\nabla| A||=C|\nabla H|=0,
$$

we conclude that $\Sigma$ is isoparametric and by Lawson's result $\Sigma$ must be $S^{1}(r) \times \mathbb{R}^{n-1}$. 


\section{VOLUME ESTIMATES FOR SUBMANIFOLDS With WEIGHTED MEAN CURVATURE OF AT MOST LINEAR GROWTH}

In this section, we show some volume estimates and prove the second main result of this paper.

Let us consider $D_{r} \subset M$ as the level set

$$
D_{r}=\{x \in M ; 2 \sqrt{f}<r\},
$$

and $V(r)$ the volume of $\bar{D}_{r}$.

Theorem 4.1. Let $\left(M^{n}, g, e^{-f} d v\right)$ be a complete non-compact smooth measure metric space, with $f: M \rightarrow \mathbb{R}$ a proper function on M. If $|\nabla f|^{2} \leq f$ and $\Delta_{f} f+f \leq a_{2} r^{2}+a_{1} r+a_{0}$ on $D_{r}$ for all $r>0$, where $a_{0}, a_{1}, a_{2}$ are constants, then $\operatorname{Vol}_{f}(M)<\infty$ and for $\varepsilon>0$ arbitrary

$$
V(r) \leq C e^{\varepsilon\left(a_{2} r^{2}+a_{1} r+a_{0}\right)+\frac{r^{2}}{4 e^{\varepsilon}}} .
$$

Proof. Let us define

$$
I(t)=\frac{1}{t^{\kappa(r)}} \int_{\bar{D}_{r}} e^{-\frac{f}{t}} d v
$$

for all $t>0$, where $\kappa(r)=a_{2} r^{2}+a_{1} r+a_{0}$. Since $\mathrm{f}$ is proper, $I$ is well defined.

Computing the derivative of $I$, we get

$$
I^{\prime}(t)=t^{-\kappa(r)-1} \int_{\bar{D}_{r}} e^{-\frac{f}{t}}\left(\frac{f}{t}-\kappa(r)\right) d v .
$$

On the other hand

$$
\begin{aligned}
\int_{\bar{D}_{r}} \operatorname{div}\left(e^{-\frac{f}{t}} \nabla f\right) d v & =\int_{\bar{D}_{r}} e^{-\frac{f}{t}}\left(\Delta f-\frac{|\nabla f|^{2}}{t}\right) d v \\
& \leq \int_{\bar{D}_{r}} e^{-\frac{f}{t}}\left(|\nabla f|^{2}-f+\kappa(r)-\frac{|\nabla f|^{2}}{t}\right) d v \\
& \leq \int_{\bar{D}_{r}} e^{-\frac{f}{t}}\left(\frac{(t-1)}{t} f-f+\kappa(r)\right) d v, \quad t \geq 1 \\
& =\int_{\bar{D}_{r}} e^{-\frac{f}{t}}\left(\kappa(r)-\frac{f}{t}\right) d v \\
& =-I^{\prime}(t) t^{\kappa(r)+1} .
\end{aligned}
$$

Therefore, we have

$$
I^{\prime}(t) \leq-t^{-\kappa(r)-1} \int_{\bar{D}_{r}} \operatorname{div}\left(e^{-\frac{f}{t}} \nabla f\right) d v
$$

For every $r$ such that $\frac{r^{2}}{4}$ is a regular value of $\mathrm{f}, D_{r}$ has smooth boundary. By the Stokes theorem, we get

$$
\begin{aligned}
I^{\prime}(t) & \leq-t^{-\kappa(r)-1} \int_{\partial D_{r}}\left\langle e^{-\frac{f}{t}} \nabla f, \frac{\nabla f}{|\nabla f|}\right\rangle d v \\
& \leq-t^{-\kappa(r)-1} \int_{\partial D_{r}} e^{-\frac{f}{t}}|\nabla f| d v \leq 0 .
\end{aligned}
$$

Integrating $I^{\prime}(t)$ over $t$, from 1 to $e^{\varepsilon}$, where $\varepsilon>0$ is arbitrary, we obtain $I\left(e^{\varepsilon}\right) \leq I(1)$, that is

$$
e^{-\varepsilon \kappa(r)} \int_{\bar{D}_{r}} e^{-\frac{f}{e^{\varepsilon}}} d v \leq \int_{\bar{D}_{r}} e^{-f} d v
$$


By the monotone convergence theorem, the inequality above holds for any $r>0$. Since $2 \sqrt{f} \leq r$ on $\bar{D}_{r}$, we have

$$
e^{-\varepsilon \kappa(r)} e^{-\frac{r^{2}}{4 e^{\varepsilon}}} \int_{\bar{D}_{r}} d v \leq \int_{\bar{D}_{r}} e^{-f} d v
$$

Moreover,

$$
\int_{\bar{D}_{r}} e^{-f} d v-\int_{\bar{D}_{r-1}} e^{-f} d v=\int_{\bar{D}_{r} \backslash \bar{D}_{r-1}} e^{-f} d v \leq e^{-\frac{(r-1)^{2}}{4}} \int_{\bar{D}_{r}} d v
$$

Combining (4.4) and (4.5), we get

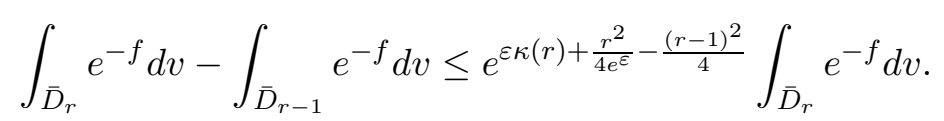

Since $\kappa(r)=a_{2} r^{2}+a_{1} r+a_{0}$, there exist $r_{0} \in \mathbb{R}$ such that for $r \geq r_{0}$ and $\varepsilon_{0}$ sufficiently small

$$
e^{\varepsilon_{0}\left(a_{2} r^{2}+a_{1} r+a_{0}\right)+\frac{r^{2}}{4 e^{\varepsilon} 0}-\frac{(r-1)^{2}}{4}}<e^{-r} .
$$

From (4.6), we have

$$
\int_{\bar{D}_{r}} e^{-f} d v \leq \frac{1}{1-e^{-r}} \int_{\bar{D}_{r-1}} e^{-f} d v
$$

Then for any integer $\mathrm{N}$

$$
\int_{\bar{D}_{r_{0}+N}} e^{-f} d v \leq\left(\prod_{i=0}^{N} \frac{1}{1-e^{-r_{0}-i}}\right) \int_{\bar{D}_{r_{0}-1}} e^{-f} d v<\infty,
$$

which implies that $\int_{M} e^{-f} d v<+\infty$. Moreover, from (4.4) we obtain

$$
e^{-\varepsilon \kappa(r)} e^{-\frac{r^{2}}{4 e^{\varepsilon}}} \int_{\bar{D}_{r}} d v \leq i n t_{\bar{D}_{r}} e^{-f} d v \leq \int_{M} e^{-f} d v<\infty .
$$

Therefore

$$
V(r) \leq C e^{\varepsilon \kappa(r)+\frac{r^{2}}{4 e^{\varepsilon}}}
$$

Remark 4.1. An immediate consequence is that under the same hypothesis of Theorem 4.1, for $\varepsilon=0$ we have the following volume estimate

$$
V(r) \leq C e^{\frac{r^{2}}{4}} .
$$

In the following result, we obtain a volume estimate for submanifolds with weighted mean curvature of at most linear growth. In particular, we obtain an explicit estimate for the volume of self-expanders and translating solitons.

Corollary 4.2. Let $\Sigma^{n} \subset \mathbb{R}^{n+p}$ be a complete submanifold such that

$$
\left|\vec{H}_{f}\right| \leq a_{1} r+a_{0} \text { on } \Sigma \cap B_{r}(0), \forall r>0
$$

where $a_{0}, a_{1} \geq 0$. If $\Sigma$ is properly immersed on $\mathbb{R}^{n+p}$, then

$$
V\left(B_{r}(0) \cap \Sigma\right) \leq C e^{\varepsilon\left(\frac{a_{1} r^{2}+a_{0} r+n}{2}\right)+\frac{r^{2}}{4 e^{\varepsilon}}}
$$

for $\varepsilon>0$ arbitrary. 
Proof. Let us verify that $f$ satisfies the conditions of Theorem 4.1, Indeed

$$
f-|\nabla f|^{2}=\frac{\left|x^{\perp}\right|^{2}}{4} \geq 0
$$

and

$$
\begin{aligned}
\Delta_{f} f+f & =\frac{n}{2}+\langle\vec{H}, \bar{\nabla} f\rangle-|\nabla f|^{2}+f \\
& =\frac{n}{2}+\left\langle\overrightarrow{H_{f}}-(\bar{\nabla} f)^{\perp},(\bar{\nabla} f)^{\perp}\right\rangle-|\nabla f|^{2}+f \\
& =\frac{n}{2}+\left\langle\overrightarrow{H_{f}},(\bar{\nabla} f)^{\perp}\right\rangle .
\end{aligned}
$$

Therefore

$$
\Delta_{f} f+f \leq \frac{a_{1} r^{2}+a_{0} r+n}{2}
$$

on $\Sigma \cap B_{r}(0)$. Since $\Sigma$ is properly immersed, it follows that $\mathrm{f}$ is proper on $\Sigma$. Applying Theorem 4.1, we obtain $\int_{\Sigma} e^{-f}<\infty$ and

$$
V\left(B_{r}(0) \cap \Sigma\right) \leq C e^{\varepsilon\left(\frac{a_{1} r^{2}+a_{0} r+n}{2}\right)+\frac{r^{2}}{4 e^{\varepsilon}}}
$$

for $\varepsilon>0$ arbitrary.

Using the corollary above, we will prove Theorem 1.4 . Proof of Theorem 1.4. From Corollary 4.2, we get that (i) implies (ii). To prove that (ii) implies (iii), we provide the following estimate

$$
\begin{aligned}
\int_{\Sigma} e^{\frac{-|x|^{2}}{4}} d v & \leq \sum_{j=1}^{\infty} \int_{\Sigma \cap B_{j} \backslash B_{j-1}} e^{\frac{-|x|^{2}}{4}} d v \\
& \leq \sum_{j=1}^{\infty} e^{\frac{-(j-1)^{2}}{4}} V\left(\Sigma \cap B_{j}\right) \\
& \leq C \sum_{j=1}^{\infty} e^{\frac{-(j-1)^{2}}{4}} e^{\bar{a}_{2} r^{2}+\bar{a}_{1} r+\bar{a}_{0}} \\
& =C \sum_{j=1}^{\infty} e^{\frac{\left(4 \bar{a}_{2}-1\right) j^{2}+\left(2+4 \bar{a}_{1}\right) j+\left(4 \bar{a}_{0}-1\right)}{4}} .
\end{aligned}
$$

Since $\bar{a}_{2}<\frac{1}{4}$, the right side of the inequality converges. Therefore

$$
\int_{\Sigma} e^{\frac{-|x|^{2}}{4}} d v<\infty
$$

Finally, we prove that (iii) implies $(i)$. Indeed, suppose $\Sigma$ is not proper. Then there exists $r_{0}$ such that $\bar{B}_{r_{0}} \cap \Sigma$ is not compact on $\Sigma$. Thus, for a positive constant $a$, there exists a sequence $\left\{p_{k}\right\}$ on $\bar{B}_{r_{0}} \cap \Sigma$ such that $d_{\Sigma}\left(p_{k}, p_{j}\right) \geq a$. Therefore, $B^{\Sigma}\left(p_{k}, \frac{a}{2}\right) \cap B^{\Sigma}\left(p_{j}, \frac{a}{2}\right)=\emptyset$, where $B^{\Sigma}$ is the geodesic ball on $\Sigma$. Choosing $0<a<\min \left\{2 r_{0}, \frac{n}{\left(2 a_{1}+1\right) r_{0}+a_{0}}\right\}$, for all $p \in B^{\Sigma}\left(p_{k}, \frac{a}{2}\right)$

$$
|p| \leq\left|p-p_{k}\right|+\left|p_{k}\right| \leq d_{\Sigma}\left(p, p_{k}\right)+\left|p_{k}\right| \leq 2 r_{0}
$$

which implies that $B^{\Sigma}\left(p_{k}, \frac{a}{2}\right) \subset B\left(0,2 r_{0}\right)$ for all $k$.

Since $\Sigma$ is a CWMC hypersurface, for all $p \in \Sigma \cap B\left(0,2 r_{0}\right)$

$$
\begin{aligned}
|\vec{H}|(p) & \leq\left|\overrightarrow{H_{f}}(p)\right|+\frac{\left|p^{\perp}\right|}{2} \\
& \leq\left(2 a_{1}+1\right) r_{0}+a_{0} .
\end{aligned}
$$


Considering $r_{k}: B^{\Sigma}\left(p_{k}, \frac{a}{2}\right) \rightarrow \mathbb{R}$, where $r_{k}(x)=\left|x-p_{k}\right|$, we have

$$
\begin{aligned}
\Delta r_{k}^{2} & =2 n+\left\langle\vec{H}, \nabla r_{k}^{2}\right\rangle \\
& \geq 2 n-2|\vec{H}| r_{k} \\
& \geq 2 n-2\left(a_{0}+\left(2 a_{1}+1\right) r_{0}\right) r_{k} .
\end{aligned}
$$

By the divergence theorem, since $a \leq \frac{n}{\left(2 a_{1}+1\right) r_{0}+a_{0}}$, for all $0<r<\frac{a}{2}$

$$
\begin{aligned}
\int_{B^{\Sigma}\left(p_{k}, r\right)}\left[2 n-2 a_{0} r_{k}-2\left(2 a_{1}+1\right) r_{0} r_{k}\right] d v & \leq \int_{B^{\Sigma}\left(p_{k}, r\right)} \Delta r_{k}^{2} d v \\
& =\int_{\partial B^{\Sigma}\left(p_{k}, r\right)}\left\langle\nabla r_{k}^{2}, \nu\right\rangle d v \\
& \leq 2 r A(r)
\end{aligned}
$$

where $\nu$ is the outward normal vector of $\partial B^{\Sigma}\left(p_{k}, r\right)$ and $A(r)$ the area of $\partial B^{\Sigma}\left(p_{k}, r\right)$. Using the co-area formula, we obtain

$$
\begin{aligned}
\int_{0}^{r}\left[n-a_{0} s-\left(2 a_{1}+1\right) r_{0} s\right] A(s) d s & =\int_{0}^{r} \int_{d_{\Sigma}\left(x, p_{k}\right)=s}\left[n-a_{0} r_{k}-\left(2 a_{1}+1\right) r_{0} r_{k}\right] d v \\
& =\int_{B^{\Sigma}\left(p_{k}, r\right)}\left[n-a_{0} r_{k}-\left(2 a_{1}+1\right) r_{0} r_{k}\right] d v \\
& \leq r A(r) .
\end{aligned}
$$

Therefore,

$$
\left(\frac{n}{r}-a_{0}-\left(2 a_{1}+1\right) r_{0}\right) \leq \frac{V^{\prime}(r)}{V(r)}
$$

Integrating from $\varepsilon>0$ to $r$, we obtain

$$
\log \left(\frac{r}{\varepsilon}\right)^{n}-\left(a_{0}+\left(2 a_{1}+1\right) r_{0}\right)(r-\varepsilon) \leq \log \frac{V(r)}{V(\varepsilon)} .
$$

Thus

$$
r^{n} e^{-\left(a_{0}+2\left(a_{1}+1\right) r_{0}\right)(r-\varepsilon)} \frac{V(\varepsilon)}{\varepsilon^{n}} \leq V(r)
$$

Since

$$
\lim _{\varepsilon \rightarrow 0} \frac{V(\varepsilon)}{\varepsilon^{n}}=\omega_{n}
$$

for any $0<r \leq \frac{a}{2}$, we have

$$
V(r) \geq r^{n} \omega_{n} e^{-\left(a_{0}+2\left(a_{1}+1\right) r_{0}\right) r} .
$$

Finally, considering that $B^{\Sigma}\left(p_{k}, \frac{a}{2}\right) \cap B^{\Sigma}\left(p_{j}, \frac{a}{2}\right)=\emptyset$ for $k \neq j, B^{\Sigma}\left(p_{k}, \frac{a}{2}\right) \subset B\left(0,2 r_{0}\right)$ for all $k$, and the inequality obtained above, we have

$$
\begin{aligned}
\int_{\Sigma} e^{-\frac{|x|^{2}}{4}} d v & \geq \sum_{k=1}^{\infty} \int_{B^{\Sigma}\left(p_{k}, \frac{a}{2}\right)} e^{-\frac{|x|^{2}}{4}} d v \\
& \geq e^{-r_{0}^{2}} \sum_{k=1}^{\infty} V\left(\frac{a}{2}\right)=+\infty
\end{aligned}
$$

which is a contradiction.

An immediate consequence of the theorem above is as follows. 
Corollary 4.3. For any complete n-dimensional CWMC hypersurface $\Sigma$ in $\mathbb{R}^{n+1}$, the following statements are equivalent:

(i) $\Sigma$ properly immersed on $\mathbb{R}^{n+1}$;

(ii) There exist constants $a_{2}, a_{1}, a_{0}$, with $a_{2}<\frac{1}{4}$, and $C>0$ such that

$$
V\left(B_{r}(0) \cap \Sigma\right) \leq C e^{a_{2} r^{2}+a_{1} r+a_{0}} ;
$$

(iii) $\int_{\Sigma} e^{-f}<\infty$.

\section{Application of the main Results}

To prove an application of the main theorems of this paper, we need the following lemma:

Lemma 5.1. Let $\Sigma \subset \mathbb{R}^{n+1}$ be a complete CWMC hypersurface properly embedded such that $H-\lambda>0$. If

$$
\lambda\left(\operatorname{tr} A^{3}(H-\lambda)+\frac{|A|^{2}}{2}\right) \leq 0,
$$

then $\int_{\Sigma}|A|^{2} e^{-f}<\infty$.

Proof. Let us compute $\mathcal{L}(\log (H-\lambda))$,

$$
\begin{aligned}
\Delta \log (H-\lambda) & =\operatorname{div}(\nabla \log (H-\lambda)) \\
& =\operatorname{div}\left(\frac{1}{H-\lambda} \nabla(H-\lambda)\right) \\
& =\frac{1}{H-\lambda} \Delta(H-\lambda)-\frac{1}{(H-\lambda)^{2}} \mid \nabla\left(H-\left.\lambda\right|^{2} .\right.
\end{aligned}
$$

From Lema 2.2, we get

$$
\begin{aligned}
\mathcal{L}(\log (H-\lambda)) & =\frac{1}{H-\lambda} \mathcal{L}(H-\lambda)-|\nabla \log (H-\lambda)|^{2} \\
& =\frac{1}{2}-|A|^{2}+\frac{\lambda}{2(H-\lambda)}-|\nabla \log (H-\lambda)|^{2} .
\end{aligned}
$$

Considering $\eta \in C_{0}^{\infty}(\Sigma)$ and integrating the equation above we have,

$$
\begin{aligned}
\int_{\Sigma} \eta^{2}\left(|A|^{2}-\frac{1}{2}-\frac{\lambda}{2(H-\lambda)}+|\nabla \log (H-\lambda)|^{2}\right) e^{-f} & =-\int_{\Sigma} \eta^{2} \mathcal{L}(\log (H-\lambda)) e^{-f} \\
& =\int_{\Sigma}\left\langle\nabla \eta^{2}, \nabla \log (H-\lambda)\right\rangle e^{-f} \\
& \leq \int_{\Sigma}\left(|\nabla \eta|^{2}+\eta^{2}|\nabla \log (H-\lambda)|^{2}\right) e^{-f}
\end{aligned}
$$

Therefore,

For $\lambda<0$, we get

$$
\int_{\Sigma} \eta^{2}|A|^{2} e^{-f} \leq \int_{\Sigma}\left(|\nabla \eta|^{2}+\frac{\eta^{2}}{2}+\frac{\lambda \eta^{2}}{2(H-\lambda)}\right) e^{-f}
$$

$$
\int_{\Sigma} \eta^{2}|A|^{2} e^{-f} \leq \int_{\Sigma}\left(|\nabla \eta|^{2}+\frac{\eta^{2}}{2}\right) e^{-f}
$$

Let us consider a sequence $\eta_{k} \in C_{0}^{\infty}(\Sigma)$, such that $\eta_{k}=1$ in $B_{k}^{\Sigma}(p), \eta_{k}=0$ in $\Sigma \backslash B_{k+1}^{\Sigma}(p)$ and $\left|\nabla \eta_{k}\right| \leq 1$ for every $k$. By the monotone convergence theorem and the condition (iii) in Corollary 4.3, we can conclude the proof for this case.

When $\lambda>0$, by the hypothesis we have

$$
\frac{\lambda}{H-\lambda} \leq-\frac{2 \lambda \operatorname{tr} A^{3}}{|A|^{2}} \leq 2|\lambda||A| \leq|\lambda|\left(\frac{|A|^{2}}{\delta}+\delta\right)
$$


Therefore,

$$
\int_{\Sigma}\left(1-\frac{|\lambda|}{2 \delta}\right) \eta^{2}|A|^{2} e^{-f} \leq \int_{\Sigma}\left(|\nabla \eta|^{2}+\left(1+\frac{|\lambda| \delta}{2}\right) \eta^{2}\right) e^{-f}
$$

for any $\delta>0$. For $\delta$ sufficiently large, we get

$$
\int_{\Sigma} \eta^{2}|A|^{2} e^{-f} \leq \frac{1+\frac{|\lambda| \delta}{2}}{1-\frac{|\lambda|}{2 \delta}} \int_{\Sigma}\left(|\nabla \eta|^{2}+\frac{\eta^{2}}{2}\right) e^{-f}
$$

Using the same argument as before, we conclude the proof of the lemma.

As a corollary of Theorem 1.3 and the lemma above, we prove Cheng-Wei classification theorem.

Corollary 5.2. Let $\Sigma \subset \mathbb{R}^{n+1}$ be a complete properly embedded CWMC hypersurface. If $H-\lambda \geq 0$ and $\lambda\left(\operatorname{tr} A^{3}(H-\lambda)+\frac{|A|^{2}}{2}\right) \leq 0$, then $\Sigma$ must be either a hyperplane or $S_{r}^{k}(0) \times \mathbb{R}^{n-k}, 1 \leq k \leq n$.

Proof. To prove this corollary, we only need to see that the condition (iii) is satisfied in Theorem 1.3 . Hence

$$
\frac{1}{k^{2}} \int_{B_{2 k}^{\Sigma}(p) \backslash B_{k}^{\Sigma}(p)}|A|^{2} e^{-f} \leq \frac{1}{k^{2}} \int_{\Sigma}|A|^{2} e^{-f} .
$$

From Lemma 5.1, $\int_{\Sigma}|A|^{2} e^{-f}<\infty$. Therefore, when $k \rightarrow 0$, we have

concluding the proof.

$$
\frac{1}{k^{2}} \int_{B_{2 k}^{\Sigma}(p) \backslash B_{k}^{\Sigma}(p)}|A|^{2} e^{-f} \rightarrow 0
$$

\section{REFERENCES}

[AR18] Hilário Alencar and Adina Rocha, Stability and geometric properties of constant weighted mean curvature hypersurfaces in gradient ricci solitons, Annals of Global Analysis and Geometry (2018), 1-21.

[CM12] Tobias H Colding and William P Minicozzi, Generic mean curvature flow $i$; generic singularities, Annals of Mathematics (2012), 755-833.

[CVZ19] Xu Cheng, Matheus Vieira, and Detang Zhou, Volume growth of complete submanifolds in gradient ricci solitons with bounded weighted mean curvature, arXiv preprint arXiv:1909.05724 (2019).

[CW18] Qing-Ming Cheng and Guoxin Wei, Complete $\lambda$-hypersurfaces of weighted volume-preserving mean curvature flow, Calculus of Variations and Partial Differential Equations 57 (2018), no. 2, 32.

[CZ13] Xu Cheng and Detang Zhou, Volume estimate about shrinkers, Proceedings of the American Mathematical Society 141 (2013), no. 2, 687-696.

$\left[\mathrm{DX}^{+} 13\right]$ Qi Ding, YL Xin, et al., Volume growth eigenvalue and compactness for self-shrinkers, Asian Journal of Mathematics 17 (2013), no. 3, 443-456.

[Gua18] Qiang Guang, Gap and rigidity theorems of $\lambda$-hypersurfaces, Proceedings of the American Mathematical Society 146 (2018), no. 10, 4459-4471.

[Hei17] Steven Heilman, Symmetric convex sets with minimal gaussian surface area, arXiv preprint arXiv:1705.06643 (2017).

[Hui] Gerhard Huisken, Local and global behaviour of hypersurfaces moving by mean curvature. differential geometry: partial differential equations on manifolds (los angeles, ca, 1990), 175-191, Proc. Sympos. Pure Math, vol. 54.

[Hui90] , Asymptotic-behavior for singularities of the mean-curvature flow, Journal of Differential Geometry 31 (1990), no. 1, 285-299.

[Law69] H Blaine Lawson, Local rigidity theorems for minimal hypersurfaces, Annals of Mathematics (1969), $187-197$.

[Rim14] Michele Rimoldi, On a classification theorem for self-shrinkers, Proceedings of the American Mathematical Society 142 (2014), no. 10, 3605-3613.

[TZ17] Ditter Tasayco and Detang Zhou, Uniqueness of grim hyperplanes for mean curvature flows, Archiv der Mathematik 109 (2017), no. 2, 191-200.

Saul Ancari, Instituto de Matemática e Estatística, Universidade Federal Fluminense, 24210-201 Niterói, Rio DE JANEIRO, BRAZIL

E-mail address: sa_ancari@id.uff.br

Igor Miranda, Instituto de Matemática e Estatística, Universidade Federal Fluminense, 24210-201 Niterói, Rio DE JANEIRO, BRAZIL

E-mail address: igor_miranda@id.uff.br 